\title{
Some things change, some don't. An exploration of Seyfert galaxy luminosity changes over a generation
}

\author{
Hartmut Winkler* \\ Department of Physics, University of Johannesburg \\ E-mail: hwinkler@uj.ac.za
}

In the 1980's some of the first systematic photometric studies established that Seyfert galaxies are variable on typical timescales of months. In some instances the luminosity, and even some of the spectral characteristics have changed dramatically over the years. In contrast, other AGN also classified as broad-line Seyferts have proved to be much more photometrically stable. The paper presents re-observations of several AGN obtained at SAAO in 2016-2017. These are used with other recent data to highlight a potential correlation between the spectral characteristics and the variability pattern. The significance of this correlation is briefly explored.

5th Annual Conference on High Energy Astrophysics in Southern Africa

4-6 October 2017

University of the Witwatersrand (Wits), South Africa

\footnotetext{
* Speaker.
} 


\section{Introduction}

Seyfert galaxies were identified as a specific class of objects about 75 years ago [1]. Their extraordinary nuclear brightness and spectral peculiarities were soon recognised to be the consequence of the release of gravitational energy from matter in rapid motion towards and around a supermassive black hole. [2]

The nuclei of Seyfert galaxies have long been known to be variable [3]. Long-term light curves require decades to establish, so little more could be determined about the photometric behaviour of AGN at the time. The quantity and quality of available photometric data however improved substantially in years to come. Doroshenko and collaborators [4] published a 15-year light curve of Akn 120 that shows that the nucleus has varied by as much as a magnitude in the $U$-band during this timespan. Attempts to identify patterns or periodicity in AGN variations (e.g. [5] [6]) were usually not successful, though some recent discoveries point to possible cyclical behaviour [7]. In contrast to the fast flickering observed in Seyfert galaxies at high energy bands, the optical nuclear luminosities change more slowly, with clear variations usually only observable on time scales of about a month.

Some AGN have now been identified that experience dramatic spectral transitions from one emission class to another (e.g. Seyfert 1 to Seyfert 1.8). One documented case of such a 'changing look' AGN is NGC 2617 [8]. Another such object, Mkn 926, is discussed in the next section.

While it is firmly expected that any AGN will display variability if observed sufficiently often, there is no clarity on what the range of these variations is likely to be, and whether this range depends on AGN properties such as the average luminosity or spectral peculiarities. This paper explores the viability of performing such an analysis based on just two photometric data points and statistical arguments.

\section{Long-term variability in AGN}

This investigation applies the following approach to quantify variations. It utilises a sample of objects for which just two photometric measurements exist. The initial measurement $i$ is combined with a second measurement $j$, taken many years later. If the time scale between these readings is large enough, the readings may be considered as two random sampling points in the lifespan of a particular AGN.

The parameter $\rho_{i j}$ is now introduced to quantify the variation measured as follows:

$$
\rho_{i j}=2 \Delta f / f_{a v, i j}=\left(f_{j}-f_{i}\right) /\left(f_{i}+f_{j}\right)
$$

where $f_{i}$ and $f_{j}$ are two arbitrary flux measurements and $f_{a v, i j}$ is their average. When measured repeatedly for new $f_{i}$ and $f_{j}$ collected at different epochs, the $\rho_{i j}$ would follow a gaussian distribution with a mean value of $\rho=0$ and a standard deviation $\sigma(\rho)$ approaching $\sigma(f) / f_{a v}$.

So, while it is of course not possible to pronounce on the variability range of individual objects based on two brightness measurements only, a histogram of the $\rho_{i j}$ determined for a large sample of similar AGN should produce a gaussian curve of width representative of the sample. If the gaussian curves for two samples made of different AGN subclasses exhibit different widths, this would show that individual AGN subclasses also have unequal variation ranges. 


\subsection{Mkn 926}

Mkn 926 (also referred to as MCG -2-58-22) is an unusual broad-line AGN at $z=0.0469$. Its Seyfert nature was first reported by Ward et al. [9], who identified it as the optical counterpart of the x-ray source 2A 2302-088. They also highlighted the following features in the discovery spectrum: (a) the exceptional width of the broad lines, (b) the asymmetric nature of the Balmer lines, with a blue hump evident for $\mathrm{H} \beta$ and (c) the complete absence Fe II emission.

It was soon recognised as a photometrically variable AGN. De Ruiter and Lub [10] produced photometric measurements of Mkn 926 at roughly annual intervals from December 1979 to December 1982 in the Walraven photometric system. Using the conversion formula supplied by these authors, their measurements may be converted to Johnson $V$-magnitudes (which are in agreement with some $U B V$ observations from the same epoch by Mallama [11]). Between 7 and 10 Sep 1985 the magnitude averaged $V=13.77$ and $V=13.64$ through the $17 \operatorname{arcsec}$ and 24 arcsec diameter apertures respectively [12].

Winkler et al [5] measured Mkn 926 to be a bright $V=13.68$ (with a $30 \operatorname{arcsec}$ diameter aperture) on 1 Sep 1986, but within a year the $V$-magnitude had dropped by half a magnitude. As the $V$-band flux contains a substantial fraction of light originating from the host galaxy (rather than the distinctively blue nucleus), the brightness drop would have been even more dramatic if a $U$ magnitude or a smaller aperture measurement had been used for the earlier date. A general fading of the nucleus ensued in the years to 1994 (with some 'flickering'), which was monitored in that and a follow-up paper [6].

While the historical photometric measurements utilised a wider range of aperture sizes, it is possible to convert these to a standard aperture size by assuming a standard exponential disk profile determined by matching near-simultaneous $15 \mathrm{arcsec}, 20 \mathrm{arcsec}$ and $30 \mathrm{arcsec}$ data from Winkler et al. [5]. A light curve of estimated $10 \operatorname{arcsec}$ aperture $V$-magnitudes covering the period 1977-1994 is presented in Fig. 1.

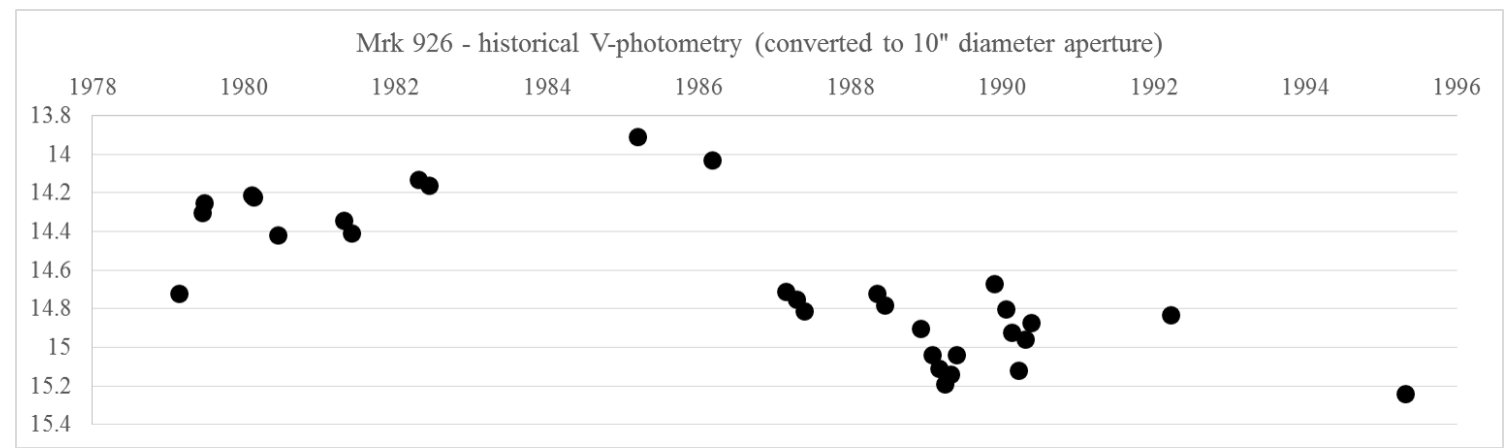

Figure 1: The Mrk $926 V$-band historical light curve. Data points come from [13], [10], [11], [12], [5] and [6]

In summary, three distinct phases can be defined for the evolution of Mrk 926: (a) a bright phase beginning before 1978 that culminated in maximum luminosity in 1985-1986; (b) a phase from 1986 to 1994 with an underlying fading of the nuclear continuum and broad lines; (c) a 
Table 1: Range of $U$-band variations determined from other long-term photometry of AGN

\begin{tabular}{lllccl}
\hline Object & $z$ & Ref. & aperture & Date range & $\sigma(\rho)$ \\
\hline 3C 120 & 0.0330 & {$[14]$} & $14^{\prime \prime}$ & $2002-2008$ & 0.22 \\
3C 120 & 0.0330 & {$[14]$} & $27^{\prime \prime}$ & $1997-2008$ & 0.23 \\
NGC 4151 & 0.0033 & {$[15]$} & $27^{\prime \prime}$ & $1968-1998$ & 0.51 \\
Mkn 6 & 0.0188 & {$[16]$} & $27^{\prime \prime}$ & $1970-2001$ & 0.24 \\
\hline
\end{tabular}

comparatively faint phase, characterised by complex broad line profiles and an unusual narrow line spectrum.

\subsection{Other Seyfert galaxies with long monitoring records}

Table 1 presents four long-term published photometric data sets of well-known AGN, all covering multiple epochs, including what appear to be both bright and faint phases.

The standard deviations of the U-band fluxes determined from these datasets are presented in the last column of Table 1. These values were determined by converting the $U$-magnitudes to fluxes, and calculating the standard deviation $\sigma(\rho)$ of these fluxes. While this procedure fails to take into account that the data was not sampled at random (in fact data tends to be bunched into multiple short-term time spans that coincide with allocated telescope time), it is expected that $\sigma(\rho)$ provides a reasonable estimate of the true standard deviation of the flux of an uninterrupted, long-term light curve of a particular AGN.

It is also necessary to note that, as the photons detected through a typical aperture include a constant component of light from the host galaxy generated outside the AGN, the true nuclear variability in general exceeds the amount measured through aperture photometry. This host galaxy contamination effect can however be minimised through the use of U-filter data, where the ratio of nuclear photons to host galaxy photons is at a maximum [5].

\section{Observations}

Photometric observations were carried out with the $1.0 \mathrm{~m}$ telescope at the South African Astronomical Observatory (SAAO) Sutherland site. The data presented here was obtained during four observing weeks. The first of these was in October 1997, when the imaging device was a TEK8 CCD. The final three observing weeks were in December 2016, and in June and September 2017 with an STE4 CCD. All images presented here were obtained through a Johnson U filter.

Details of the observed objects and the two observation dates are given in Table 2. Integration times ranged between $600 \mathrm{~s}$ and $1200 \mathrm{~s}$, depending on object brightness and sky conditions. The sky was photometric in at least one of the epochs of observation. This enabled the determination of photometric magnitudes of the targets as well as other stars on the frame that were then used as comparison stars for observations made in partially cloudy conditions. Calibrations were achieved through regularly nightly observations of photometric standard stars from the list of Menzies et al [17]. 
Table 2: List of Seyfert galaxies observed

\begin{tabular}{|c|c|c|c|c|}
\hline 00) & Object & $z$ & Dates of observation & $\rho_{12}$ \\
\hline $00: 10: 10-04: 42: 40$ & kn 93 & 0295 & $1997 / 10 / 10,2016 / 12 / 21$ & 0.047 \\
\hline & & & & \\
\hline 6 & & & & \\
\hline & & & & \\
\hline 03:11 & nU & & 20 & +0.066 \\
\hline 17 & CTS & & & \\
\hline $04: 2$ & $\mathrm{HE}$ & & 19 & -0.036 \\
\hline $04: 4$ & TS & & & +0.034 \\
\hline 04: & IRA & & & +0 \\
\hline & $\mathrm{y}$ & & & +0.259 \\
\hline & & & & \\
\hline & CTS & & & -0 \\
\hline & & & & \\
\hline 06: & $\mathrm{RXC}$ & & & +0.143 \\
\hline & & & 1997 & +0.060 \\
\hline $21: 19$ & & & & -0.087 \\
\hline 21: & CTS & 67 & $1997 /$ & -0.088 \\
\hline & & & & -0.130 \\
\hline 22: & Q 22 & 55 & 19 & -0.327 \\
\hline & & & & +0.032 \\
\hline $22: 5$ & $\mathrm{RXJ} 2$ & 60 & $/ 18$ & +0.170 \\
\hline & Mkn & & & +0.189 \\
\hline 23:59:11 -04:07:37 & IC 1524 & 0.0192 & 1997/10/10, 2017/06/17 & +0.187 \\
\hline
\end{tabular}

Collected frames were corrected for bias and chip spatial sensitivity fluctuations through the use of twilight flatfield recordings in the standard manner. Readings on pixels within the apertures affected by cosmic rays were replaced by suitably averaged values from neighbouring pixels.

The use of the U-filter has the advantage that the images accentuate the blue Seyfert nuclei and suppress the flux contribution from the host galaxy, which is very faint in that band. Fluxes were measured by integrating the counts inside a ring positioned so that its centre coincides with the AGN nucleus, alternatively the position of a comparison star. Aperture sizes varied from frame to frame, depending on the seeing and the projected galaxy size on the image. Typically aperture diameters were chosen that corresponded to 2-3 times the seeing disk diameter.

Fluxes in the apertures were then scaled to achieve as good a match in the comparison star fluxes. The resulting AGN fluxes, labeled $f_{1}$ for the 1997 and $f_{2}$ for the 2016/17 values respectively, were then used to determine $\rho_{12}$ for each object.

\section{Results}

Figure 2 plots the the $\rho_{12}$-values listed in the last column of Table 2 as a function of redshift 
z. It also displays the range in variations for Mkn 926 and the three Seyferts comprehensively monitored in previous studies reported in Table 1.



Figure 2: $\rho$ distribution for the observed AGN as a function of redshift $z$. Also indicated are the range in $\sigma(\rho)$ (about $\rho=0$ ) found in the data sets published in the literature by other researchers (see Table 1) for NGC 4151, Mkn 6, 3C 120 and Mkn 926.

The plot suggests that the variation range for the more distant (and thus usually more luminous) objects is smaller than for the more nearby ones. The standard deviation $\sigma(\rho)$ is 0.237 for the subsample of 10 objects with $z<0.05$, and 0.144 for the 13 further AGN. This corresponds to an $F$-statistic of 2.728 , implying that, when applying the statistical $F$-test, the probability is greater than $90 \%$ that the more distant sample has an intrinsically smaller variation range.

3C 120 and Mkn 6 vary in a range that is consistent with the observed sample. The variation range of the very nearby NGC 4151 however exceeds the observed sample average considerably.

The most striking change in luminosity over the 20-year period was observed in CTS A32.01, where in 2017 the $U$-band luminosity was only $28 \%$ of the 1997 luminosity. Frames illustrating this object during the two epochs are presented in Fig. 3.

It is cautioned here that the higher $\sigma(\rho)$ for the low redshift sample is entirely due to CTS A32.01, and that there would be no statistical dependence of $\sigma(\rho)$ on redshift if that object was to be left out of the analysis.

\section{Discussion and Conclusion}

This investigation suggests that in the long term the nuclear luminosity of most AGN varies about a mean luminosity with an amplitude described by a standard variation of about 20\%. Abnormally large brightness changes are however possible, and luminous or faint phases can persist for many years in objects referred to as 'changing look' Seyferts. 


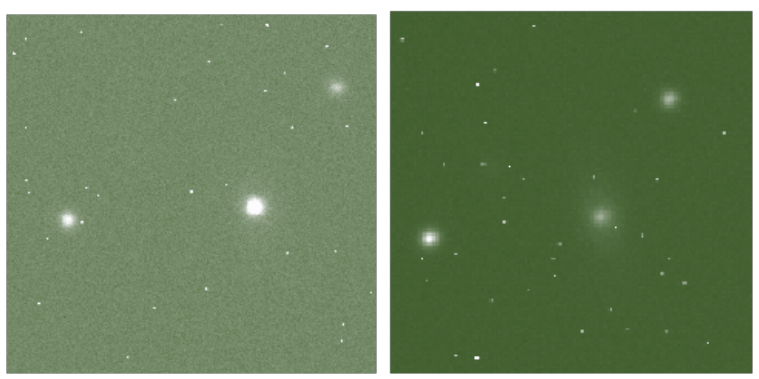

Figure 3: $U$ image of CTS A32.01 in 1997 (left) and in 2017 (right), with two nearby comparison stars to its left and top right in the images.

The luminosity drop experienced by CTS A32.01 pinpoints this as a potential 'changing look' AGN. Only one past spectrum has been identified in the available literature, recorded on 10 Mar 2003 as part of the 6dF survey [18]. In that spectrum the broad Balmer lines are not very strong, and the object would probably be classified as a Seyfert 1.8. CTS A32.01 was presumably brighter during 1997 as well as at the time of its discovery a few years before that.

Although this study was carried out with a relatively small sample including a variety of Seyfert galaxy spectral sub-classes and a large range of luminosities, it indicates that the procedure is feasible, and should be tested on different samples. In particular, inter-comparisons should be made of homogeneous samples representative of specific spectral sub-classes (such as the I Zw 1 type objects, whose luminosities seem relatively stable [5]). While the lower-redshift Seyferts studied here on average varied more than their more distant counterparts, this finding cannot be generalised unless it can be supported by further analysis using a much bigger sample.

This present work however suggests that the process described here could be profitably applied to the numerous AGN with just occasional brightness measurements, such as are often encountered in vast data sets such as the one associated with the Sloan Digital Sky Survey [19].

\section{Acknowledgments}

The author thanks the South African Astronomical Observatory for the use of its telescope and facilities, and its technical, scientific and support team for their able assistance when required.

\section{References}

[1] C. K. Seyfert, Nuclear Emission in Spiral Nebulae., Astrophys. J. 97 (1943) 28.

[2] M.-P. Véron-Cetty and P. Véron, A catalogue of quasars and active nuclei: 13th edition, Astron. Astrophys. 518 (2010) A10.

[3] M. V. Penston, M. J. Penston, R. A. Selmes, E. E. Becklin and G. Neugebauer, Broadband optical and infrared observations of Seyfert galaxies, Mon. Not. R. astr. Soc. 169 (1974) 357-393.

[4] V. T. Doroshenko and V. M. Lyuty, UBV photometry of the Seyfert Galaxy Akn 120 in 1974-1999, Astronomy Letters 25 (1999) 771-780. 
[5] H. Winkler, I. S. Glass, F. van Wyk, F. Marang, J. H. S. Jones, D. A. H. Buckley et al., Variability studies of Seyfert galaxies. I - Broad-band optical photometry, Mon. Not. R. astr. Soc. 257 (1992) 659-676.

[6] H. Winkler, The extinction, flux distribution and luminosity of Seyfert 1 nuclei derived from $U B V(R I)_{C}$ aperture photometry, Mon. Not. R. astr. Soc. 292 (1997) 273.

[7] Y.-R. Li, J.-M. Wang, Z.-X. Zhang, K. Wang, Y.-K. Huang, K.-X. Lu et al., A Possible 20-Year Periodicity in Long-term Variations of the Nearby Radio-Quiet Active Galactic Nucleus Ark 120, ArXiv e-prints (2017), [1705.07781].

[8] V. L. Oknyansky, C. M. Gaskell, N. A. Huseynov, V. M. Lipunov, N. I. Shatsky, S. S. Tsygankov et al., The curtain remains open: NGC 2617 continues in a high state, Mon. Not. R. astr. Soc. 467 (2017) 1496-1504.

[9] M. J. Ward, A. S. Wilson, M. V. Penston, M. Elvis, T. Maccacaro and K. P. Tritton, Optical identifications of extragalactic X-ray sources, Astrophys. J. 223 (1978) 788-797.

[10] H. R. de Ruiter and J. Lub, Optical variability of Seyfert nuclei. I - VBLUW photometry, Astron. Astrophys. Suppl. 63 (1986) 59-69.

[11] A. D. Mallama, UBV Photometry of Seyfert Galaxy Nuclei, Journal of the American Association of Variable Star Observers (JAAVSO) 12 (1983) 69.

[12] M. Hamuy and J. Maza, UBVRI photometry of active galaxies. I - Observations, Astron. Astrophys. Suppl. 68 (1987) 383-396.

[13] V. T. Doroshenko and V. Y. Terebizh, UBVR photometry of Seyfert galaxies, Astrophysics 17 (1981) 358-362.

[14] V. T. Doroshenko, S. G. Sergeev, Y. S. Efimov, S. A. Klimanov and S. V. Nazarov, Comparison of the $X$-ray and optical variabilities in the Seyfert galaxy 3C 120, Astronomy Letters 35 (2009) 361-374.

[15] V. M. Lyuty and V. T. Doroshenko, A new activity cycle of NGC4151: Evidence for the existence of an accretion disk, Astronomy Letters 25 (1999) 341-350.

[16] V. T. Doroshenko, Photometric activity of the Seyfert galaxy Markarian 6 from UBV observations in 1970-2001, Astron. Astrophys. 405 (2003) 903-908.

[17] J. W. Menzies, A. W. J. Cousins, R. M. Banfield and J. D. Laing, UBV(RI) standard stars in the Eand F-regions and in the Magellanic Clouds - a revised catalogue, South African Astronomical Observatory Circular 13 (1989) 1-13.

[18] D. H. Jones, M. A. Read, W. Saunders, M. Colless, T. Jarrett, Q. A. Parker et al., The 6dF Galaxy Survey: final redshift release (DR3) and southern large-scale structures, Mon. Not. R. astr. Soc. 399 (2009) 683-698.

[19] B. Abolfathi, D. S. Aguado, G. Aguilar, C. Allende Prieto, A. Almeida, T. Tasnim Ananna et al., The Fourteenth Data Release of the Sloan Digital Sky Survey: First Spectroscopic Data from the Extended Baryon Oscillation Spectroscopic Survey and from the Second Phase of the Apache Point Observatory Galactic Evolution Experiment, Astrophys. J. Suppl. 235 (2018) 42. 